\title{
Integration of hepatitis B vaccination into rural African primary health care programmes
}

\author{
B D Schoub, S Johnson, J M McAnerney, N Blackburn, M C Kew, J P McCutcheon, N D Carlier
}

\begin{abstract}
Objective-To determine the efficacy of hepatitis $B$ vaccine when added to the routine expanded programme on immunisation under field conditions in rural Africa.

Design-Infants were immunised according to two schedules - an early schedule at birth, 3 months, and 6 months and a later schedule to correspond with routine vaccination in the expanded programme on immunisation at 3 months, $4 \frac{1}{2} 2$ months, and 6 months.
\end{abstract}

Setting-Venda, northern Transvaal, South Africa, a self governing region of 7460 square kilometres varying from rural villages to small towns.

Subjects - The 1989 birth cohort of Venda.

Main outcome measures-Coverage for hepatitis $B$ vaccine at first, second, and third doses; serological assessment of vaccine efficacy by prevalence of antibodies to hepatitis B surface antigen in infants who had completed the three dose course of immunisation; antibodies to hepatitis $B$ core antigen to determine if natural infection occurred.

Results-Vaccine coverage for hepatitis B dropped sharply from $99 \%$ to $53 \%$ to $39 \%$ for the first, second, and third doses respectively. In contrast, vaccine coverage was maintained at $97-99 \%$ for the three doses of poliomyelitis vaccine. Serological evaluation of vaccine efficacy showed that only $3.5 \%$ of recipients of all three doses failed to develop antibodies to hepatitis B surface antigen. Only 6.6\% of vaccine recipients were vaccinated according to either the early or later schedules whereas $93.4 \%$ received their doses of vaccine at intervals beyond the limits of either of the planned schedules. There was, however, no significant difference in seroconversion to the surface antigen between the "unscheduled" or scheduled groups or those who were vaccinated according to the early or late schedules. The pattern of prevalence of antibodies to hepatitis B core antigen, which showed a sharp fall in children aged over 7 months, suggested that the antibodies were acquired passively rather than by active infection.

Conclusions -Supplementation of the present expanded programme on immunisation with hepatitis $B$ vaccine in rural Africa is fraught with difficulties. However, the vaccine was effective within a fairly wide spacing of dosage. Adding hepatitis $B$ vaccine to diphtheria, tetanus, and pertussis as a tetravalent vaccine is proposed as a means of effectively integrating it into the expanded programme on immunisation in Third World settings.

\section{Introduction}

The World Health Organisation, ${ }^{12}$ as well as other organisations, ${ }^{34}$ have called for the addition of hepatitis $B$ virus vaccine to the six existing vaccines in the expanded programme on immunisation for routine immunisation of all infants where hepatitis $B$ virus is endemic, especially the Far East and sub-Saharan Africa. Far Eastern countries such as Taiwan and Singapore have successfully implemented routine hepatitis B immunisation programmes. ${ }^{56}$ In Africa a hepatitis B vaccine trial in 156 Senegalese infants has shown both short term efficacy and long term persistence of antibodies, ${ }^{78}$ and in The Gambia a cooperative programme between the Gambian government, the International Agency for Research on Cancer, and the Medical Research Council of the United Kingdom was highly effective in introducing the vaccine into the country's national expanded programme on immunisation. ${ }^{9}$ In most of rural Africa, however, conditions are far removed from the idealised circumstances encountered in these two African studies or in Asian countries where mass immunisation has already been implemented, and no study has yet examined the complexities of nationwide introduction of a new vaccine such as hepatitis $B$ vaccine into the expanded programme on immunisation in Africa.

During 1989 a year long campaign supplementing the routine expanded programme on immunisation with hepatitis B vaccine was carried out in the Republic of Venda, a self governing region of some 7460 square kilometres in the far north of South Africa. With a population of some 500000 at a density of 69 people per square kilometre, Venda consists almost entirely of black communities living in conditions varying from rural agricultural settlements to small towns. As in other rural African communities hepatitis B endemicity is high, and a 1986 study of 29312 adult black male mineworkers of rural origin from throughout southern Africa found that the hepatitis B surface antigen carrier rate ranged from $5 \cdot 5 \%$ to $14 \cdot 0 \%$, with that from Venda being $8 \cdot 7 \% .^{10}$ Primary health care facilities are excellent in Venda and an unpublished WHO expanded programme on immunisation cluster survey carried out in March 1988 found vaccine coverage rates for children aged 12-23 months for BCG; third dose of polio and diphtheria, tetanus, and pertussis; and measles of $98 \%, 93 \%$, and $91 \%$ respectively. Thus, Venda was thought to be a suitable region to initiate routine supplementation of the expanded programme on immunisation with a gift of hepatitis B vaccine derived from plasma (H-B Vax; Merck Sharp and Dohme). Furthermore, a highly motivated health authority gave enthusiastic support before and during the project.

\section{Materials and methods}

PRIMARY HEALTH CARE PROGRAMME IN VENDA

In 1989 the Department of Health in Venda provided primary health care programmes, including weekly child health clinics to the community through 57 fixed health care delivery points (three hospitals, 
two health centres, two maternity centres, and 50 clinics). The department also operated three GOBI teams, which are mobile child health clinics servicing 274 visiting points on a six week cycle. (GOBI is the acronym for growth monitoring, oral rehydration, breast feeding, and immunisation, which are health techniques propounded by WHO and Unicef to achieve the goal of health for all by the year 2000. $\left.{ }^{11}\right)$ At child health clinics, growth monitoring of children under 5 years, health education, and vaccination are carried out.

\section{SEROLOGICAL SURVEY OF WOMEN ATTENDING} ANTENATAL CLINICS

Between December 1988 and February 1989 serum samples from 1296 women attending either various rural antenatal clinics in Venda or the major regional hospital, Tshilidzini Hospital, were tested for hepatitis B markers. Specimens were initially tested for antibodies to hepatitis B surface antigen and antibodies to hepatitis B core antigen, and if both were negative or if they were positive for surface antigen (with or without a positive result for core antigen) no further tests were done. Specimens positive for antibodies to core antigen alone were further tested for surface antigen, and if this was positive, hepatitis $\mathrm{B}$ e antigen was also tested for.

\section{ADMINISTRATION OF VACCINE}

For about three months before the start of the immunisation programme at the beginning of 1989 considerable publicity was provided in the form of information pamphlets distributed to all health care workers, numerous meetings, and radio messages to the population by well known people including the local minister of health. Acceptance by both health care workers and the population seemed to be excellent, and awareness throughout the region was confirmed by inspections during the course of the campaign.

Hepatitis $\mathrm{B}$ vaccine was administered in $10 \mathrm{~g}$ doses by intramuscular injection into the anterolateral aspect of the thigh. To fit in with delivery of vaccine in the expanded programme on immunisation two schedules of administration were followed: at birth (up to 14 days), at 3 months (80-100 days), and at 6 months (170-190 days) of age; and for babies who could not be reached at birth, at 3 months (80-100 days), $4 \frac{1 / 2}{2}$ months (125-145 days), and 6 months (170-190 days) of age. Infants who were not reached within the limits of the above schedules were classed as "unscheduled." Vaccine was administered both by fixed clinics and by mobile GOBI teams.

Vaccine cold chain monitoring cards accompanied vaccine batches to fixed vaccination points and those with mobile teams and ensured that vaccines were maintained below $10^{\circ} \mathrm{C}$ throughout the study.

POSTIMMUNISATION SEROLOGICAL SURVEY OF INFANTS

Capillary blood specimens were taken from 863 infants who had received all three doses of hepatitis $B$ vaccine from one professional nursing sister from a heelprick using Autoclix lancets (Boehringer Mannheim, Mannheim, Germany) and collected into microtainer capillary blood-serum separation tubes (Becton Dickinson, Rutherford, New Jersey). The specimens were kept at $4^{\circ} \mathrm{C}$ in a domestic refrigerator until their weekly shipment by carrier to the National Institute for Virology for testing. Only infants who had received the full course of three doses of vaccine 28 or more days before the blood sample was taken were included in the study. Each specimen was accompanied by the name of the infant, date of birth, sex, parents' names and addresses, clinic attended, and hepatitis B vaccine dates. All 863 specimens were tested for antibodies to hepatitis B surface antigen, and in 683 there was also sufficient serum for testing for antibodies to core antigen, but there was insufficient serum in most specimens for any additional tests.

All blood specimens, both antenatally from the mothers and from the infants, were taken only after obtaining informed consent from the mothers. The study was approved by the human ethics committee of the University of Witwatersrand.

\section{SEROLOGY}

All hepatitis $B$ virus markers were tested by radioimmunoassay (Abbott Laboratories, Chicago, Illinois), and antibodies to hepatitis $\mathrm{B}$ surface antigen were measured in $\mathrm{mIU} / \mathrm{ml}$ using the WHO reference preparation as a standard. A positive titre was defined as having $\geqslant 10 \mathrm{mIU}$ of antibodies. Data were analysed statistically using either a $\chi^{2}$ test or Fisher's exact test.

\section{Results}

In the study of mothers attending antenatal clinics, $3 \cdot 5 \%(46 / 1296)$ were found to be carriers of hepatitis B surface antigen $(95 \%$ confidence interval $2 \cdot 5 \%$ to $4.6 \%$ ), four of whom were also positive for hepatitis B e antigen; $53 \cdot 1 \%(688)(50 \cdot 3 \%$ to $55 \cdot 8 \%)$ were positive for antibodies to surface or core antigen, or both.

Immunisation coverage for hepatitis $\mathrm{B}$ vaccine dropped sharply from $99 \%$ for the first dose to $39 \%$ for the third dose, in contrast to $86 \%$ for BCG, and $97-99 \%$ for polio (table I). Of the 863 completely immunised infants evaluated serologically, only $30(3.5 \%)$ failed to respond to the vaccine. Analysis of the data accompanying the specimens showed that few of the infants received their doses of vaccine within the limits of either of the schedules: thus $1 \cdot 6 \%$ (14 of 863 ) were vaccinated according to the early schedule and $5.0 \%$ (43) according to the late schedule; $93 \cdot 4 \%$ (806) fell into the "unscheduled" group. This unscheduled group received vaccine as follows: first dose 0-224 (median 27) days after birth; second dose 1-215 (58) days after the first dose; the third dose 8-182 (49) days after the second dose. There was, however, no significant difference in the number who seroconverted to the surface antigen between the early schedule and the late schedule groups, $(13 / 14(93 \%) v$ $42 / 43(98 \%) ; p=0 \cdot 43$, Fisher's exact test), or between the scheduled and unscheduled groups $(55 / 57(97 \%)$ $v 778 / 806(97 \%) ; \mathrm{p}=0 \cdot 10$, Fisher's exact test), or between those who were immunised in fixed clinics and by mobile teams $(625 / 652(96 \%) v 208 / 211(99 \%)$; $\mathrm{p}=0.06, \chi^{2}$ test $)$. This lack of difference was also noted in the subgroup of infants who had samples taken after 8 months, when most passively acquired maternal antibodies would have disappeared (early schedule 6/7 $(86 \%) v$ late schedule $38 / 39$ (98\%); $\mathrm{p}=0 \cdot 28$, Fisher's

TABLE II - Measured antibody responses to hepatitis B surface antigen in infants vaccinated according to different schedules. Figures are numbers (percentages) of children receiving all three doses of hepatitis $B$ vaccine

\begin{tabular}{|c|c|c|c|c|}
\hline & $>10 \mathrm{mIU}$ & $10-99 \mathrm{mIU}$ & $100-999 \mathrm{mIU}$ & $1000 \mathrm{mIU}$ \\
\hline Early schedule & $1(7)$ & $1(7)$ & $3(21)$ & $9(64)$ \\
\hline Late schedule & $1(2)$ & $6(14)$ & $11(26)$ & $25(58)$ \\
\hline Total & $2(4)$ & $7(12)$ & $14(25)$ & $34(60)$ \\
\hline Scheduled & $2(4)$ & $7(12)$ & $14(25)$ & $34(60)$ \\
\hline Unscheduled & $28(4)$ & $66(8)$ & $320(40)$ & $392(49)$ \\
\hline Total & $30(4)$ & $73(9)$ & $334(39)$ & $426(49)$ \\
\hline Clinic & $27(4)$ & $58(9)$ & $262(40)$ & $305(47)$ \\
\hline Mobile team & $3(1)$ & $15(7)$ & $72(34)$ & $121(57)$ \\
\hline Total & $30(3)$ & $73(9)$ & $334(39)$ & $426(49)$ \\
\hline Aged $<8$ months & $6(3)$ & $16(8)$ & $102(50)$ & $79(39)$ \\
\hline Aged $\geqslant 8$ months & $24(4)$ & $57(9)$ & $232(35)$ & $347(53)$ \\
\hline Total & $30(4)$ & $73(9)$ & $334(39)$ & $426(49)$ \\
\hline
\end{tabular}




\begin{tabular}{lc}
\hline $\begin{array}{l}\text { Age } \\
\text { (months) }\end{array}$ & $\begin{array}{c}\text { No (\%) positive for } \\
\text { antibodies to hepatitis } \\
\text { B core antigen }\end{array}$ \\
\hline 4 & $1 / 12(8)$ \\
5 & $4 / 33(12)$ \\
6 & $8 / 50(16)$ \\
7 & $9 / 77(12)$ \\
8 & $1 / 95(1)$ \\
9 & $1 / 105(1)$ \\
10 & $3 / 93(3)$ \\
11 & $1 / 98(1)$ \\
12 & $0 / 120$ \\
\hline
\end{tabular}

exact test; scheduled 44/46 (96\%) $v$ unscheduled $594 / 616(96 \%) ; \mathrm{p}=0.68$, Fisher's exact test; clinic $447 / 468(96 \%) v$ mobile teams $191 / 194(99 \%) ; \mathrm{p}=0 \cdot 07$, $\chi^{2}$ test). Similarly, no apparent differences were observed in the degree of antibody response to surface antigen measured in mIU between all these categories of vaccine recipients (table II).

The prevalence of antibodies to hepatitis B core antigen decreased sharply in children aged over 7 months (table III): 13\% (22/172) of specimens from infants aged 4-7 months were positive compared with $1 \%(6 / 511)$ from infants aged 8 months or more $(\mathrm{p}<0.001)$.

In three newborn babies a self limiting osteomyelitis of the femur developed after inoculation, and two minor injection abscesses were also seen after the first injection. No other side effects from the vaccine were reported.

\section{Discussion}

Sub-Saharan Africa is a vast reservoir of hepatitis $B$ virus infection, and routine immunisation programmes need to be implemented urgently. With the present state of primary health care programmes in most of the continent, however, adding another vaccine to prevent a disease that is largely invisible during infancy and childhood requires considerable planning to devise effective strategies. Three major difficulties need to be considered before hepatitis B vaccine can be incorporated as a seventh vaccine in the expanded programme on immunisation in rural Africa Firstly, the cost of the vaccine must be reduced, and vaccine manufacturers and perhaps also sponsoring bodies must strive to bring the cost below $\$ 1$ per dose to these countries. ${ }^{3}$ The second and third fundamental difficulties relate to the need for wide flexibility of dosage schedules and also the means of ensuring maintenance of satisfactory coverage for the second and third doses.

In the initial evaluations of the efficacy of hepatitis B vaccine in neonates and infants between 1981 and 1985 seroconversion rates varying from $64 \%$ to $100 \%$ were achieved. ${ }^{12}$ In most of these trials vaccine schedules of 0,1 , and 6 months or 0,1 , and 2 months were used to conform to the dosage schedules recommended for adult immunisation and to administer the first dose as soon as possible after birth to interrupt perinatal transmission of hepatitis B virus. Adding an extra vaccine to the present six in the expanded programme on immunisation would, however, necessitate integration in ways that strengthen existing programmes, and therefore timing of vaccine doses would need to comply with existing schedules.

Administration of hepatitis $B$ vaccine at $0,2,4$, and 9 months in The Gambia ${ }^{9}$ and three injections at monthly intervals with a booster a year later in Senegal, ${ }^{8}$ were highly effective in short term and long term seroresponses. Nevertheless, two major difficulties remain in regard to field conditions in rural African communities. Firstly, there is a difficulty in complying with WHO recommendations for the first dose to be given as soon as possible after birth because most births do not take place in hospital or clinics and access for the neonatal dose is difficult. Secondly, administration of all three doses within reasonable limits of the recommended vaccine schedules is often difficult to implement in rural African conditions, where distances to clinics are often great and mobile outreach clinics are impeded by poor road conditions. Indeed, in our study $93.4 \%$ of the infants evaluated serologically fell into the "unscheduled" group who received their three doses of vaccine at widely varying times. However, unlike the Far East where perinatal transmission plays a key part in maintaining carriage of chronic hepatitis B virus, ${ }^{5}$ in southern Africa horizontal transmission later in infancy is of greater importance than perinatal spread. Thus in rural Kangwane in the eastern Transvaal perinatal infection was found to be uncommon,,$^{13}$ and in Namibia most carriers had been infected after the age of 11 months, with only $1 \%$ being infected before the age of 6 months. ${ }^{14}$ It was consequently decided to implement a later schedule of vaccination at $3,4 \frac{1}{2}$, and 6 months in addition to the more conventional early schedule of 0 , 3 , and 6 months. A similar later schedule $(3,5$, and 11 months) used for a mass immunisation programme in Italy achieved a $96 \cdot 3 \%$ seroresponse rate. ${ }^{15}$

Evaluation of the sample of 863 fully immunised infants showed that $96.5 \%$ were positive for antibodies to hepatitis B surface antigen. No significant differences in seroresponse rates were observed between those receiving vaccine in the early or later schedules, whether a defined schedule was followed or vaccine doses were given at widely variable intervals, or whether vaccine was administered by fixed clinics or by mobile GOBI teams. Unfortunately, few capillary specimens were available with sufficient serum to test for surface antigen to assess protection, but tests for antibodies to core antigen could be carried out on 683 of the 863 specimens and the distribution of the 28 positive results, occurring predominantly in infants aged 7 months or less and declining sharply thereafter, is consistent with passively acquired maternal antibodies rather than with active infection. The low prevalence of antibodies to core antigen could also be explained by infection with the variant of hepatitis B virus 2 described in Senegal ${ }^{16}$ which produces a positive result for surface antigen but a negative result for core antigen, although these variants have not yet been observed in this part of Africa. Botha et al similarly found passively acquired maternal antibodies persisting up to the age of 7-12 months. ${ }^{14}$ Integration of hepatitis B vaccine into routine immunisation programmes can thus be highly effective in rural Africa even within wide limits of dosage schedules. Starting immunisation could also be delayed by two or three months to fit in with the expanded programme on immunisation wherever there would be difficulties in administering the neonatal dose.

Of great concern was the high dropout rate after the first dose. The health region had had excellent vaccine coverage on previous WHO cluster surveys and during the term of this study maintained coverage for polio 1 , 2 , and 3 (and presumably also for diphtheria, tetanus, and pertussis 1, 2, and 3) at $97-99 \%$. Acceptance and awareness after extensive educational and publicity efforts throughout the region was particularly high, and this was borne out by the $99 \%$ coverage for the first dose. Yet in spite of this there was obviously a breakdown in complete implementation of the vaccination programme at the periphery after the first dose of hepatitis $B$ vaccine had been administered. There were a number of possible reasons for this. Firstly, the novel hepatitis B vaccine was introduced and distributed differently from the vaccines in the expanded programme on immunisation with which primary health care workers were familiar, resulting in recurring shortages at vaccination points. Thus, although infants would be present at scheduled times, coverage for hepatitis B was handicapped by the need for an additional catch up visit. This was further compromised by mobile clinic visits being spaced at six week intervals. Confusion also arose with regard to whether infants fell into the planned early or late schedule group when the first dose had been delayed for a few weeks, and this occasionally resulted in failure to complete either schedule. In some cases health care workers and mothers complained about the extra injection for hepatitis, and mothers were also requested to 
return on a separate occasion for the hepatitis $B$ immunisation. Defaulting did not seem to be a contributing factor to the sharp drop off of hepatitis B vaccine coverage beyond the first dose. In the 1988 vaccination coverage survey defaulters were few. In addition, clinics can identify them by duplicate road to health cards, and defaulters' homes are visited by clinic sisters. More importantly, hepatitis B vaccine was not always available when children did attend clinics.

In either event the high dropout rate in an otherwise excellent primary health care programme emphasises the difficulties in introducing a new vaccine to routine expanded programme on immunisation programmes and the need to devise strategies to minimise disruption. Previous studies have shown that diphtheria, tetanus, and pertussis, BCG, and hepatitis $B$ vaccines may effectively be administered simultaneously. ${ }^{17} \mathrm{~A}$ tetravalent vaccine for diphtheria, tetanus, pertussis, and hepatitis B, with all four constituents mixed into the same phial, would ensure that hepatitis B vaccine coverage would at least reach that of diphtheria, tetanus, and pertussis.

This project was made possible by a generous gift of vaccine from Merck Sharp and Dohme and a grant from the Poliomyelitis Research Foundation of South Africa. We thank Sister Molete and the medical and nursing staff of the department of health services of the Republic of Venda for their outstanding help and dedication, Mr K O'Connell of the National Institute for Virology for excellent technical assistance, and Professor J S S Gear, head of the department of community health, University of Witwatersrand, for invaluable help in planning this project.

1 Anonymous. Global advisory group of the expanded programme on immunization. Weekly Epidemiological Record 1988;63:9-13.
2 Anonymous. Global advisory group of the expanded programme on immunization. Weekly Epidemiological Record 1990;65:5-11.

Maynard JE, Kane MA, Hadler SC. Global control of hepatitis B through accination: role of hepatitis $\mathrm{B}$ vaccine in the expanded programme on immunization. Rev Infect Dis 1989;11 suppl 3/S574-8.

4 Schoub BD, Johnson S, McAnerney. JM, Wogstaff L. Strategies for viral mon, Jir Wadee AA, ods. Health for all by the yor 2000: proceding of the syhof $\mathrm{HJ}$ We Research Council, 1988 .

5 Chen D-S, Hsu NH-M, Sung J-L, et al. A mass vaccination program in Taiwan against hepatitis $B$ virus infection in infants of hepatitis $B$ surface antigenarrier mothers. JAMA 1987;257:2597-603.

6 Goh KT, Doraisingham S, Tan KL, et al. The hepatitis B immunization programme in Singapore. Bull WHO 1989;67:65-70.

Coursaget P, Chotard J, Vincelot P, et al. Seven-year study of hepatitis B vaccine efficacy in infants from an endemic area (Senegal). Lancet 1986; $1143-5$.

8 Yvonnet $P$, Coursaget $P$, Chotard $\mathrm{J}$, et al. Hepatitis B vaccine in infants from an endemic area: long-term anti-HBs persistence and revaccination. 7 Med Virol 1987;22:315-21.

9 Hall AJ, Inskip HM, Loik F, et al. Hepatitis B vaccine in the expanded programme of immunisation: the Gambian experience. Lancet 1989; 1057-60.

10 Dusheiko GM, Brink BA, Conradic JD, Marimuthu T, Sher R. Regiona prevalence of hepatitis $B$, delta, and human immunodeficiency virus infection in southern Africa: a large population survey. Am $\mathcal{F}$ Epidemiol infection in sou $1989 \cdot 129 \cdot 138-45$.

11 United Nations Children's Fund (Unicef). The state of the world's children. New York: Oxford University Press, 1987

2 Smego RA, Halsey NA. The case for routine hepatitis B immunization in infancy for populations at increased risk. Pediatr Infect Dis $\mathcal{F}$ 1987;6:11-9.

3 Prozesky OW, Szmuness W, Stevens CE, et al. Baseline epidemiologica studies for hepatitis B vaccine trial in Kangwane. $S$ Afr Med $\mathcal{F}$ 1983;64: $891-3$.

14 Botha JF, Ritchie MJJ, Dusheiko GM, Mouton HWK, Kew MC. Hepatitis B virus carrier state in black children in Ovamboland: role of perinatal and horizontal infection. Lancet 1984;i:1210-2.

15 Piazza $M$, Da Villa G. Picciotto $L$ et al. Mass vaccination against hepatitis $B$ in infants in Italy. Lancet 1988 ;i: 1132 .

16 Coursaget $\mathrm{P}$, Bourdil C, Adamowicz P, et al. $\mathrm{HBs}$ Ag positive reactivity in man not due to hepatitis B virus. Lancet 1987;ii: 1354-8.

17 Coursaget $\mathrm{P}$, Y vonnet $\mathrm{B}$, Relyveld $\mathrm{EH}$, et al. Simultaneous administration of diphtheria-tetanus-pertussis-polio and hepatitis $B$ vaccines in a simplified immunization programme: immune response to diphtheria toxoid, tetanus immunization programme: immune response to diphtheria toxoid, tetanus
toxoid, pertussis, and hepatitis B surface antigen. Infect Immun 1986;51: toxoid

(Accepted 27 November 1990
Department of

Epidemiology and Health

Care Research, University

of Limburg, PO Box 616,

6200 MD Maastricht, The

Netherlands

Jos Kleijnen, MD, research fellow

Paul Knipschild, MD, professor of epidemiology

Gerben ter Riet, MD, research

fellow

Correspondence to:

Dr Kleijnen.

BMF 1991;302:316-23

\section{Clinical trials of homoeopathy}

\author{
Jos Kleijnen, Paul Knipschild, Gerben ter Riet
}

\section{Abstract}

Objective-To establish whether there is evidence of the efficacy of homoeopathy from controlled trials in humans.

Design-Criteria based meta-analysis. Assessment of the methodological quality of 107 controlled trials in 96 published reports found after an extensive search. Trials were scored using a list of predefined criteria of good methodology, and the outcome of the trials was interpreted in relation to their quality.

Setting-Controlled trials published world wide.

Main outcome measures - Results of the trials with the best methodological quality. Trials of classical homoeopathy and several modern varieties were considered separately.

Results-In 14 trials some form of classical homoeopathy was tested and in $\mathbf{5 8}$ trials the same single homoeopathic treatment was given to patients with comparable conventional diagnoses. Combinations of several homoeopathic treatments were tested in $\mathbf{2 6}$ trials; isopathy was tested in nine trials. Most trials seemed to be of very low quality, but there were many exceptions. The results showed a positive trend regardless of the quality of the trial or the variety of homoeopathy used. Overall, of the 105 trials with interpretable results, 81 trials indicated positive results whereas in 24 trials no positive effects of homoeopathy were found. The results of the review may be complicated by publication bias, especially in such a controversial subject as homoeopathy.
Conclusions-At the moment the evidence of clinical trials is positive but not sufficient to draw definitive conclusions because most trials are of low methodological quality and because of the unknown role of publication bias. This indicates that there is a legitimate case for further evaluation of homoeopathy, but only by means of well performed trials.

\section{Introduction}

A survey of 293 general practitioners in The Netherlands showed that $45 \%$ of them think that homoeopathic remedies are efficacious in treating upper respiratory tract infections or hay fever.' On the other hand, many doctors do not believe that homoeopathy is an efficacious treatment as it is highly implausible that infinitesimally diluted substances retain their biological effects. It is also often stated that homoeopathy has not been evaluated using modern methods - that is, controlled trials. The first argument may be true, but the second is certainly not true. Reading an article about pollen $\mathrm{C} 30$ in hay fever increased our interest in homoeopathy. ${ }^{2}$ We could not believe the positive resul (was it coincidence?) and therefore we started to search for further reports. Here we present 107 controlled trials of homoeopathy.

Homoeopathic medicine is a system developed by Samuel Hahnemann from the similia concept: "similia similibus curantur." This implies that a diluted, "potentised" agent, which (when undiluted) in healthy 